\title{
Long-term results of acetabular reconstruction using three bulk bone graft techniques in cemented total hip arthroplasty for developmental dysplasia
}

\author{
Kenichi Oe $^{1} \cdot$ Hirokazu Iida $^{1} \cdot$ Hiroshi Kawamura $^{1} \cdot$ Narumi Ueda $^{1}$. \\ Tomohisa Nakamura $^{1} \cdot$ Naofumi Okamoto $^{1} \cdot$ Yusuke Ueda $^{1}$
}

Received: 5 August 2015 / Accepted: 1 November 2015 / Published online: 14 November 2015

(C) The Author(s) 2015. This article is published with open access at Springerlink.com

\begin{abstract}
Purpose The aims of this retrospective study were to firstly introduce three practical bulk bone graft techniques based on acetabular morphology for dysplasia and secondly evaluate the long-term durability of acetabular reconstruction using those techniques combined with cemented total hip arthroplasty (THA).

Methods The study comprised 101 consecutive THAs with a minimum follow-up of ten years; these procedures were categorised as being L shape $(n=58)$, Wall $(n=33)$ and $\mathrm{D}$ shape $(n=10)$ types.

Results At the last follow-up, all bone grafts acquired trabecular reorientation, and no evidence of revision for aseptic or radiological loosening was noted.

Conclusions These bone graft techniques will be effective for improving the management of dysplasia in cemented THA by providing both acetabular reconstruction and cement containment.
\end{abstract}

Keywords Bulk bone grafts · Cemented total hip arthroplasty $\cdot$ Dysplasia $\cdot$ Acetabular morphology . Cement containment $\cdot$ Acetabular reconstruction

Kenichi Oe

oeken@hirakata.kmu.ac.jp

1 Department of Orthopaedic Surgery, Kansai Medical University, 2-5-1 Shinmachi, Hirakata City, Osaka 573-1010, Japan

\section{Introduction}

The acetabular component in total hip arthroplasty (THA) should be placed at the true hip centre because of bone stock, biomechanical factors and preventing loosening or dislocation [1-4]. However, placing the acetabular component at the true hip centre in developmental dysplasia of the hip (DDH) may lead to uncovering the dome, which would require augmentation. Some authors have described various techniques to augment acetabular deficiency, including techniques involving medial wall fracture, small cemented socket, cemented socket with bulk bone graft, uncemented socket with bulk bone graft and impaction bone grafting. Above all, bulk bone grafts have been reported to yield successful and positive long-term results.

In 1977, Harris et al. [5] reported a series of 27 dysplastic hips that were treated using femoral head grafting in THA and showed rates of loosening (46\%) and revision (20\%) after a mean of 11.8 years. It is a well-known fact that those poor results may have been due to using the primitive technique and implants with a small offset rather than using bulk bone grafts. In a recent study, Iida et al. [6] closely analysed 133 cemented THAs with acetabular bone graft, as described by Wolfgang [7], and contradicted the misleading statement by Harris et al. [5] about bulk bone grafts. According to their Kaplan-Meier analysis, they predicted that the acetabular component survival rate at 15 years was $96 \%$ and $75 \%$, with revision for aseptic and radiological loosening, respectively. Furthermore, they indicated risk factors for acetabular component loosening, including trochanteric nonunion, lateral placement of the socket and delayed trabecular re-orientation of bone graft. Concurrently, Bobak et al. [8] reported no revisions with acetabular bone graft involving 45 cemented THAs after a mean follow-up of 11 years. Akiyama et al. [9] used an evolving operative technique and predicted that the 
acetabular component survival rate at 15 years was $96 \%$ and $91 \%$, with revision for aseptic and radiological loosening as end points, respectively. The utility of bulk bone grafts for DDH remains uncontroversial, but acetabular morphology for bulk bone graft has not been discussed thus far.

We performed sophisticated acetabular reconstructions with bulk bone graft techniques, except the risk factors mentioned by Iida et al. [6]. The major changes in our study compared with the original report of Iida et al. [6] were the approach and fixation method of bulk bone graft against trochanteric nonunion and a delayed trabecular re-orientation of the bone graft. Moreover, if the centre-edge (CE) angle of the bone graft was $<0^{\circ}[10]$, minor changes were made to usage of the acetabular reinforcement device rather than using the bulk bone graft only. The aims of this study were firstly to introduce the three practical types of bulk bone graft techniques based on acetabular morphology for DDH and secondly retrospectively evaluate the long-term durability of more sophisticated acetabular reconstruction with those techniques combined with cemented THA. The hypothesis was that acetabular reconstruction with bulk bone graft techniques would be effective for improving DDH management in cemented THA.

\section{Materials and methods}

Between January 2001 and December 2013, we performed 2500 consecutive primary cemented THAs at our institution; $1453(58 \%)$ required a bone graft because of osteoarthritis (OA) with DDH, rheumatoid arthritis, rapidly destructive coxarthrosis or trauma. Of these, 101 THAs (87 patients) were performed for OA patients with DDH, with a minimum follow-up of ten years, and 25 THAs were lost to follow-up (follow-up rate, $80 \%$ ). We excluded patients (11 THAs) with the Kerboull-type acetabular reinforcement device because the $\mathrm{CE}$ angle of the bone graft was $<0^{\circ}$, and those (three
THAs) who underwent subtrochanteric shortening osteotomy for Crowe group IV hip [11]. The study population comprised 78 women and nine men, with a mean age of 57 (range, 2375) years at the time of surgery. Mean follow-up was 11 (range, 10-14) years. The study was approved by our institutional review board, and patients provided informed consent for participation and publication of findings.

Acetabular morphology was evaluated based on preoperative computed tomography (CT) planning, as described by lida et al. (Fig. 1) [12]. Briefly, CT images of the hip joint with an axial line interval of $5 \mathrm{~mm}$ were entered into the computer using original Sim-Hip simulation software, which determined diameter, location, inclination and anteversion of the acetabular component. After determining acetabular component position, the Sim-Hip provided a scout view in the anteroposterior (AP) radiograph. Accordingly, we selected three different techniques using a femoral head autograft. The techniques were classified as L shape, Wall and D shape based on diameter, height, inclination and lateral coverage, which were determined by the correlation between acetabular morphology and the planning component (Fig. 2). The Lshape type was employed in patients when the original lateral edge of the acetabulum was horizontally near the lateral edge of the acetabular component; the Wall type was employed when the original lateral edge of the acetabulum was located medially compared with the lateral edge of the acetabular component or when the base of the L shape was too small; the D-shape type was used when the original lateral edge of the acetabulum was located further laterally compared with the lateral edge of the acetabular component.

All operations were performed with the transgluteal approach using a femoral head autograft, as described by Wolfgang (Fig. 3) [7]. Firstly, the true acetabulum was reamed based on pre-operative planning. After preplacing the trial acetabular component and confirming superolateral uncovering of the dome, the resected femoral head was shaped according to the three different techniques. Cartilage of the false acetabulum
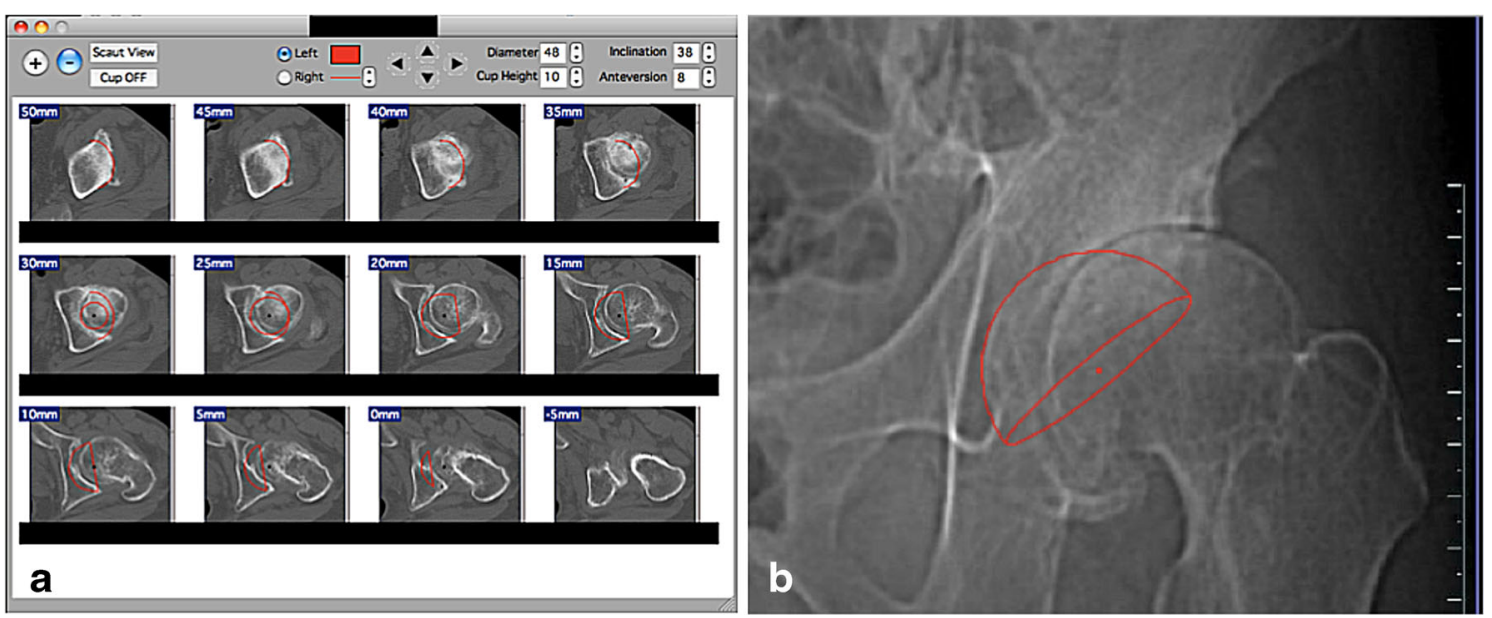

Fig. 1 A simulation software of a Sim-Hip: a preoperative computed tomography planning; b scout view of an anteroposterior radiograph 


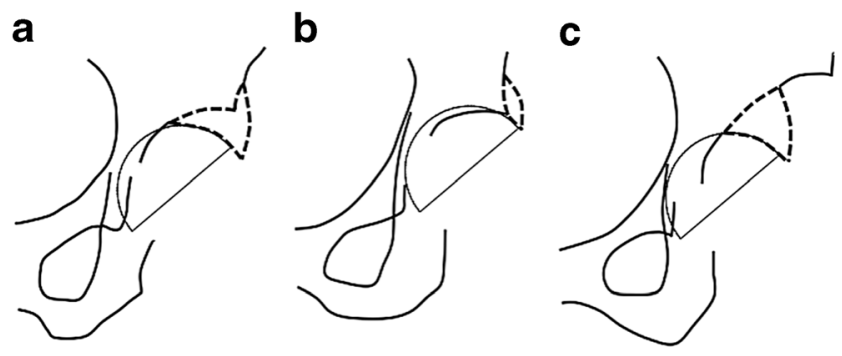

Fig. 2 The three femoral head autograft techniques: a L shape; b Wall; c D shape

was removed and shaved to expose the subchondral bone; the appropriate foundation was created at the grafting aspect of the false acetabulum. The chip bone, which was produced at the reaming of the acetabulum, was placed on the grafting aspect of the femoral head autograft and then fixed transiently with two Kirschner wires (diameter, $2.0 \mathrm{~mm}$ ). Next, the graft was fixed with bioresorbable poly-L-lactic acid screws (cancellous lag screw; $6.5 \mathrm{~mm}$ in bore diameter and $4.1 \mathrm{~mm}$ in grove diameter; Takiron, Tokyo, Japan) in 94 hips. The other seven hips were fixed using only two Kirschner wires because the graft size was small. Excessive graft bone was gradually reamed, and the covering of the dome was checked after trial acetabular component placement. Finally, two Kirschner wires were removed after acetabular component implantation. We randomly used two implant types: (1) the K-MAX CLHO flanged cup with a 22.225- $\mathrm{mm} \mathrm{ZrO}_{2}$ head and HS-3 stem in 52 hips (KYOCERA Medical, Osaka, Japan), and (2) the Charnley Elite plus cup with a $22.225-\mathrm{mm}$ Ortron 90 head and C-stem in 49 hips (DePuy International, Leeds, UK). All acetabular components were fixed with ENDURANCE bone cement (DePuy International), and all femoral components were inserted with Vacu-Mix Plus ENDURANCE bone cement (DePuy International) using a cement gun. Full weight bearing was allowed after drain removal, but using a crutch was encouraged for up to three months.

After surgery, patients were followed up at two weeks, three months, six months and annually. Blinded retrospective evaluations of the AP radiographs were performed using a ruler (Carestream Health Japan Co., Ltd., Tokyo, Japan) by two blinded orthopaedic surgeons. Two weeks after surgery, Crowe's classification [11], Hartofilakidis
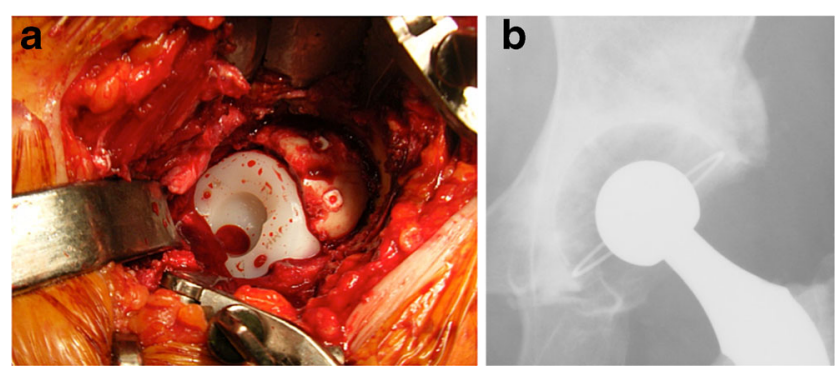

Fig. 3 a Intra-operative image and b post-operative anteroposterior radiograph in a 70-year-old woman (D-shape) classification [13], abduction angle and height from the teardrop line to the centre of the acetabular component were obtained. Graft thickness was defined as the horizontal distance between the original lateral edge of the acetabulum and the lateral edge of the bone graft. The proportion of the acetabular component covered by the bone graft was measured using the CE angle of the bone graft, which was the angle between the vertical line of the head centre and the original lateral edge of the acetabulum [10]. During follow-up, graft remodeling was assessed according to the method described by Knight et al. [14]; re-orientation of the trabecular pattern within the graft to match the normal trabecular orientation of the acetabular dome was noted. The redundant graft ratio was defined as the excessive graft (i.e., horizontal distance between the lateral edge of the acetabular component and that of the bone graft) divided by graft thickness and multiplied by 100 (\%) (Fig. 4). Graft resorption was graded as minor, moderate or major according to criteria by Gerber and Harris [15]. Resorption of $<1 / 3,1 / 3$ to $1 / 2$ and $>1 / 2$ of the graft was considered minor, moderate and major, respectively. The presence of radiolucent lines at the cement-bone interface was evaluated according to DeLee and Charnley [16]; and any evidence of acetabular component loosening was defined as complete demarcation (type 3 ) or socket migration (type 4) according to criteria by Hodgkinson et al. [17].

\section{Statistical analyses}

Survival was determined using Kaplan-Meier analysis with $95 \%$ confidence intervals (CI); endpoints were loosening and acetabular component revision. All data were analysed using one-way analysis of variance (ANOVA) with SAS version 9.2 (SAS Institute, Cary, NC, USA); a $P$ value $<0.05$ was considered significant.

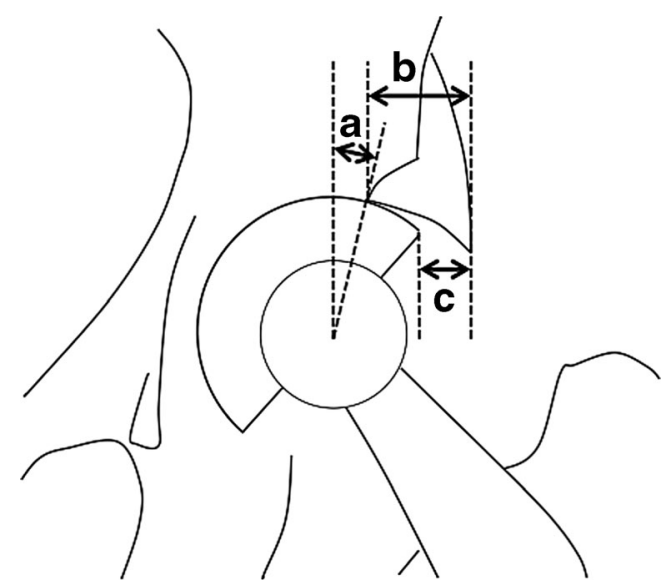

Fig. 4 a Centre-edge angle of the bone graft; $\mathbf{b}$ graft thickness; c excessive graft. Redundant graft ratio was defined as excessive graft divided by graft thickness multiplied by $100(\%)$ 
Table 1 Pre-operative patient characteristics

\begin{tabular}{llll}
\hline & L shape & Wall & D shape \\
\hline Number of hips & 58 & 33 & 10 \\
Mean age at surgery, years (range) & $58(43-74)$ & $57(23-75)$ & $57(45-65)$ \\
Gender, male:female & $05: 53$ & $05: 28$ & $00: 10$ \\
Crowe's classification I:II:III & $23: 28: 07$ & $22: 09: 02$ & $04: 05: 01$ \\
Hartofilakidis classification A:B:C & $49: 09: 00$ & $31: 02: 00$ & $05: 05: 00$ \\
Poly-L-lactic acid screw (hips) & 55 & 29 & 10 \\
Mean follow-up period, years (range) & $11(10-14)$ & $11(10-13)$ & $12(10-13)$ \\
\hline
\end{tabular}

\section{Results}

Patient demographic data are shown in Table 1 . These procedures were categorised as L shape $(n=58)$, Wall $(n=33)$, and D shape $(n=10)$ (Fig. 5). Post-operative results from the time of surgery to the last follow-up are shown in Table 2. In the Wall type, bone graft CE angle was significantly greater than that in the L- or D-shape types $(P<0.05)$. All grafts acquired trabecular re-orientation at the last follow-up. The redundant graft ratio and graft resorption in the D-shape type were significantly lower than in the L-shaped or Wall types $(P<0.05)$. No evidence of revision for aseptic and radiological loosening was noted; acetabular component rate was predicted to be $100 \%$ for both endpoints.

\section{Discussion}

In Japan, patients with hip OA have a unique aetiology. The proportion of patients who have secondary OA due to DDH is $>80 \%$ [18]. We have extensive experience in performing THA for DDH; however, DDH is a relative rare disorder in Europe and the United States. Numerous classifications of DDH have been described by some authors; the classifications by Crowe and Hartofilakidis are commonly used due to high reliability $[11,13]$. However, there are no classifications according to acetabular morphology for bulk bone grafts. In this study, we show that the three practical types of bulk bone graft techniques based on acetabular morphology can be effective for improving DDH management in cemented THA.
The aim of THA in DDH is to restore the centre of hip rotation and proximal femoral anatomy to allow optimal abductor function [19], and the acetabular component should be placed at the true hip centre by acetabular reconstruction. Many authors report excellent results of bulk bone graft for DDH in cemented THA [6, 8, 9, 20-23], and survivorship for revision of aseptic and radiological loosening is reported to be $85-100 \%$ and $82-$ $100 \%$, respectively, during a minimum follow-up of ten years. Zahar et al. [24] reported that although the midterm functional outcome of THA with acetabular bulk bone graft was satisfactory, long-term results were questionable, so the good results reported may depend on meticulous technique. No evidence of revision for aseptic and radiological loosening was noted in this study, which further supports the use of bulk bone graft for DDH. Furthermore, according to the Norwegian Arthroplasty Register, Engesaeter et al. [25] demonstrated that the risk for any revision of cementless THAs in patients with DDH was 2.7 times higher than cemented THAs. In a meta-analysis of 3488 articles, Toossi et al. [26] revealed that the estimated odds ratio for survivorship of a cemented compared to a cementless acetabular component was 1.60 (95\% confidence interval, 1.32 to $2.40 ; P=$ 0.02). According to these findings, fixation of cemented acetabular components was more reliable than that of cementless components.

Bulk bone graft techniques require some practical tools and skills, such as classification based on acetabular morphology for DDH, as mentioned. This is suitable for classifying practical bulk bone graft techniques in THA but not
Fig. 5 Post-operative anteroposterior radiographs at 10 year follow-up: a Lshape; b Wall; c $\mathrm{D}$ shape
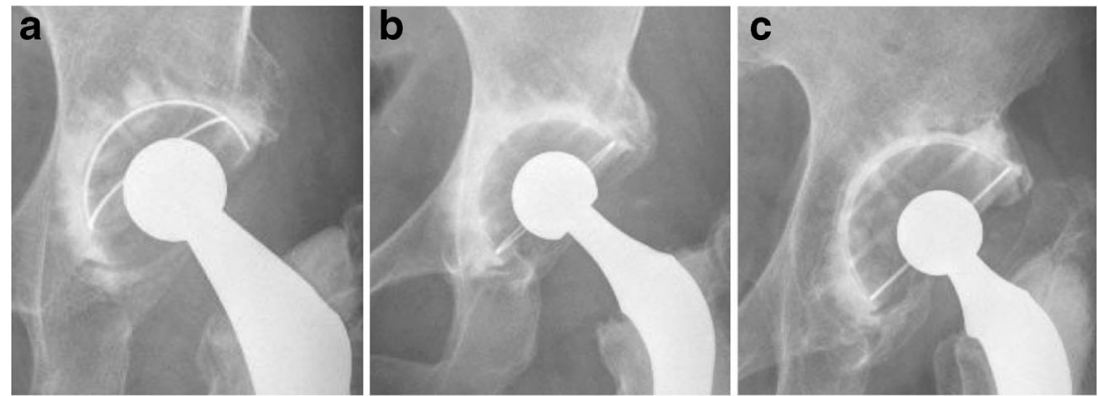
Table 2 Post-operative results from 2 weeks after surgery to last follow-up of the three different femoral head autograft techniques

\begin{tabular}{|c|c|c|c|}
\hline & L shape & Wall & D shape \\
\hline Number of hips & 58 & 33 & 10 \\
\hline Mean abduction angle, ${ }^{\circ}$ (range) & $44(38-47)$ & $43(40-48)$ & $44(39-47)$ \\
\hline Mean cup height, mm (range) & $26(15-35)$ & $26(19-37)$ & $25(16-31)$ \\
\hline Mean graft thickness at 2 weeks after surgery, mm (range) & $18(10-23)$ & $10(7-13)^{\mathrm{a}}$ & $16(11-20)$ \\
\hline Mean centre-edge angle of the bone graft at 2 weeks after surgery, ${ }^{\circ}$ (range) & $17(4-27)$ & $21(2-38)^{\mathrm{a}}$ & $18(9-26)$ \\
\hline Mean redundant graft ratio at 2 weeks after surgery, \% (range) & $30(1-41)$ & $35(1-40)$ & $7(0-10)^{\mathrm{a}}$ \\
\hline Mean redundant graft ratio at last follow-up, \% (range) & $11(1-16)$ & $10(0-14)$ & $6(0-8)$ \\
\hline Trabecular reorientation of bone graft (hips) & 58 & 33 & 10 \\
\hline Graft resorption minor:moderate:major & 40:13:05 & 20:11:02 & 10:00:00 \\
\hline Cup loosening (hips) & 0 & 0 & 0 \\
\hline
\end{tabular}

${ }^{a} P<0.05$, one-way analysis of variance

the degree of DDH [11, 13]. According to our detailed analyses, lateral resorptions of redundant bone grafts in the L-shaped and Wall types were increased compared with those in the D-shaped type because the load transfer for bone graft remodelling might be low in redundancy of the L-shaped and Wall types. The CE angle of the bone graft in the Wall type was greater (i.e. smaller graft thickness and contact area between host and graft bone), but the re-orientation of graft remodeling and long-term durability of the acetabular component were excellent in all three types. Therefore, the bone graft may play a decisive role in both acetabular reconstruction and cement containment.

There were some limitations in this study. Firstly, the current classification according to acetabular morphology and post-operative evaluations were based on AP radiographs. The resected femoral head was actually shaped according to both the scout view (2D) and simulated CT and intra-operative findings (3D). Secondly, we only evaluated the long-term durability of cemented THA in DDH with bulk bone graft, because this was not a randomised controlled study. An experimental study of acetabular exposure with DDH in the cementless acetabular component was previously conducted [27]. Although we preferred to graft the bone when the cup was covered up to $80 \%$ by the iliac bone after full preparation of the acetabulum, as described by Gerber and Harris [15], there were no strict indications for bulk bone graft; thus, our study might have included some patients who did not require a bulk bone graft. Based on the original report of Iida et al. [6], our aim was to perform acetabular reconstruction with bulk bone graft as much as possible, because complete bony containment was confirmed to be important for acetabular component survivorship [28]. Thirdly, the definition of a true hip centre is obscure and was determined intra-operatively based on the teardrop line, then assessed post-operatively as the height from the teardrop line to the centre of the acetabular component.
In summary, we obtained excellent results of more sophisticated acetabular reconstruction with bulk bone graft techniques at a minimum follow-up of ten years. Acetabular reconstruction with the three bulk bone autograft techniques (i.e. L shape, D shape, Wall) will be effective for better DDH management in cemented THA by providing both acetabular reconstruction and cement containment.

\section{Compliance with ethical standards}

Conflict of interest The authors declare that they have no conflict of interest.

Open Access This article is distributed under the terms of the Creative Commons Attribution 4.0 International License (http:// creativecommons.org/licenses/by/4.0/), which permits unrestricted use, distribution, and reproduction in any medium, provided you give appropriate credit to the original author(s) and the source, provide a link to the Creative Commons license, and indicate if changes were made.

\section{References}

1. Charnley J, Feagin JA (1973) Low-friction arthroplasty in congenital subluxation of the hip. Clin Orthop Relat Res 91:98-113

2. Johnston RC, Brand RA, Crowninshield RD (1979) Reconstruction of the hip. A mathematical approach to determine optimum geometric relationships. J Bone Joint Surg Am 61:639-652

3. Yoder SA, Brand RA, Pedersen DR, O'Gorman TW (1988) Total hip acetabular component position affects component loosening rates. Clin Orthop Relat Res 228:79-87

4. Dorr LD, Wolf AW, Chandler R, Conaty JP (1983) Classification and treatment of dislocations of total hip arthroplasty. Clin Orthop Relat Res 173:151-158

5. Harris WH, Crothers O, Oh I (1977) Total hip replacement and femoral-head bone-grafting for severe acetabular deficiency in adults. J Bone Joint Surg Am 59:752-759

6. Iida H, Matsusue Y, Kawanabe K, Okumura H, Yamamuro T, Nakamura T (2000) Cemented total hip arthroplasty with acetabular 
bone graft for developmental dysplasia. Long-term results and survivorship analysis. J Bone Joint Surg (Br) 82:176-184

7. Wolfgang GL (1990) Femoral head autografting with total hip arthroplasty for lateral acetabular dysplasia. A 12-year experience. Clin Orthop Relat Res 255:173-185

8. Bobak P, Wroblewski BM, Siney PD, Fleming PA, Hall R (2000) Charnley low-friction arthroplasty with an autograft of the femoral head for developmental dysplasia of the hip. The 10- to 15-year results. J Bone Joint Surg (Br) 82:508-511

9. Akiyama H, Kawanabe K, Iida H, Haile P, Goto K, Nakamura T (2010) Long-term results of cemented total hip arthroplasty in developmental dysplasia with acetabular bulk bone grafts after improving operative techniques. J Arthroplasty 25:716-720

10. Sugano N, Nishii T, Nakata K, Masuhara K, Takaoka K (1995) Polyethylene sockets and alumina ceramic heads in cemented total hip arthroplasty. A ten-year study. J Bone Joint Surg (Br) 77:548-556

11. Crowe JF, Mani VJ, Ranawat CS (1979) Total hip replacement in congenital dislocation and dysplasia of the hip. J Bone Joint Surg Am 61:15-23

12. Iida H, Yamamuro T, Kasai R, Matsusue Y, Matsuda Y (1993) Computed simulation for the planning of total hip replacement: Improvement of the simulation system. In: Imura $\mathrm{S}$, Akamatsu $\mathrm{N}$, Azume H, Sawai K, Tanaka S (eds) Hip Biomechanics. SpringerVerlag, Tokyo, pp 139-147

13. Hartofilakidis G, Stamos K, Karachalios T, Ioannidis TT, Zacharakis N (1996) Congenital hip disease in adults. Classification of acetabular deficiencies and operative treatment with acetabuloplasty combined with total hip arthroplasty. J Bone Joint Surg Am 78:683-692

14. Knight JL, Fujii K, Atwater R, Grothaus L (1993) Bone-grafting for acetabular deficiency during primary and revision total hip arthroplasty. A radiographic and clinical analysis. J Arthroplasty 8:371-382

15. Gerber SD, Harris WH (1986) Femoral head autografting to augment acetabular deficiency in patients requiring total hip replacement. A minimum five-year and an average seven-year follow-up study. J Bone Joint Surg Am 68:1241-1248

16. DeLee JG, Charnley J (1976) Radiological demarcation of cemented sockets in total hip replacement. Clin Orthop Relat Res 121:20-32

17. Hodgkinson JP, Shelley P, Wroblewski BM (1988) The correlation between the roentgenographic appearance and operative findings at the bone-cement junction of the socket in Charnley low friction arthroplasties. Clin Orthop Relat Res 228:105-109
18. Jingushi S, Ohfuji S, Sofue M, Hirota Y, Itoman M, Matsumoto T et al (2010) Multiinstitutional epidemiological study regarding osteoarthritis of the hip in Japan. J Orthop Sci 15:626-631

19. Kosuge D, Yamada N, Azegami S, Achan P, Ramachandran M (2013) Management of developmental dysplasia of the hip in young adults: current concepts. Bone Joint J 95:732-737

20. Busch VJ, Clement ND, Mayer PF, Breusch SJ, Howie CR (2012) High survivorship of cemented sockets with roof graft for severe acetabular dysplasia. Clin Orthop Relat Res 470:3032-3040

21. Inao S, Matsuno T (2000) Cemented total hip arthroplasty with autogenous acetabular bone grafting for hips with developmental dysplasia in adults: the results at a minimum of ten years. J Bone Joint Surg (Br) 82:375-377

22. de Jong PT, Haverkamp D, van der Vis HM, Marti RK (2006) Total hip replacement with a superolateral bone graft for osteoarthritis secondary to dysplasia: a long-term follow-up. J Bone Joint Surg (Br) 88:173-178

23. Kobayashi S, Saito N, Nawata M, Horiuchi H, Iorio R, Takaoka K (2003) Total hip arthroplasty with bulk femoral head autograft for acetabular reconstruction in developmental dysplasia of the hip. $\mathrm{J}$ Bone Joint Surg Am 85:615-621

24. Zahar A, Papik K, Lakatos J, Cross MB (2014) Total hip arthroplasty with acetabular reconstruction using a bulk autograft for patients with developmental dysplasia of the hip results in high loosening rates at mid-term follow-up. Int Orthop 38:947-951

25. Engesaeter LB, Furnes O, Havelin LI (2008) Developmental dysplasia of the hip -good results of later total hip arthroplasty: 7135 primary total hip arthroplasties after developmental dysplasia of the hip compared with 59774 total hip arthroplasties in idiopathic coxarthrosis followed for 0 to 15 years in the Norwegian Arthroplasty Register. J Arthroplasty 23:235-240

26. Toossi N, Adeli B, Timperley AJ, Haddad FS, Maltenfort M, Parvizi J (2013) Acetabular components in total hip arthroplasty: is there evidence that cementless fixation is better? J Bone Joint Surg Am 95:168-174

27. Tikhilov R, Shubnyakov I, Burns S, Shabrov N, Kuzin A, Mazurenko A, Denisov A (2015) Experimental study of the installation acetabular component with uncoverage in arthroplasty patients with severe developmental hip dysplasia. Int Orthop. doi:10. 1007/s00264-015-2951-z

28. Sarmiento A, Ebramzadeh E, Gogan WJ, McKellop HA (1990) Cup containment and orientation in cemented total hip arthroplasties. J Bone Joint Surg (Br) 72:996-1002 